\title{
WHEN EXPERIMENTS TRAVEL: CLINICAL TRIALS AND THE GLOBAL SEARCH FOR HUMAN SUBJECTS
}

\section{ADRIANA PETRYNA*}

\author{
JEFERSON FERREIRA BARBOSA ${ }^{* *}$
}

\begin{abstract}
RESUMO: com a presente resenha objetiva-se analisar e apresentar os principais temas da obra When Experiments Travel: Clinical Trials and the Global Search For Human Subjects de autoria da antropóloga Adriana Petryna. O livro aborda o tema referente à globalização dos ensaios clínicos e nos permite mergulhar em um novo olhar que remete ao contexto da interação e do conturbado encontro entre o mercado, a inovação e as necessidades de saúde pública.

PALAVRAS-CHAVE: Ensaios Clínicos Globalizados; Experimentalidade; Mercado; Saúde Pública; Inovação.
\end{abstract}

ABSTRACT: the objective of this review is to analyze and to present the main themes of the work When Experiments Travel: Clinical Trials and the Global Search For Human Subjects, it was written by anthropologist Adriana Petryna. The book addresses the issue on the globalization of clinical trials and allows us to have a new insight about the relation of market, innovation and the public health needs.

KEYWORDS: Global Clinical Trials; Experimentality; Market; Public Health; Innovation.

SUMÁRIO: Introdução; 1. Variabilidade ética; 2. Arte do desenvolvimento de medicamento; 3. O ensaio clínico global; 4. Fármacos e o direito à saúde; Conclusão.

SUMMARY: Introduction; 1. Ethical variability; 2. Arts of drug development; 3 . The global clinical trial; 4. Pharmaceuticals and the right to health; Conclusion.

\section{INTRODUÇÃO}

A autora Adriana Petryna, que também escreveu o livro Life exposed: biological citizens after Chernobyl de 2002, em 2009 publica, pela Princeton University Press, o livro When Experiments Travel: Clinical Trials and the Global Search For Human Subjects.

Artigo recebido em $1^{\circ}$.09.2010 e aprovado para publicação pelo Conselho Editorial em 29.09.2010.

* PETRYNA, Adriana. When Experiments Travel: Clinical Trials and the Global Search For Human Subjects. Princeton University Press: Princeton, 2009.

** Mestrando em Direito pela PUCRS. Bolsista Capes e Pesquisador, em nível de mestrando, no Projeto Internacional de Pesquisa Intitulado Observatório de Pesquisa e Jurisprudência de Direitos Econômicos, Sociais, Culturais e Ambientais. 
A obra investiga a indústria dos ensaios clínicos ${ }^{1}$; a forma e o contexto do seu surgimento e evolução; a descentralização e globalização da experimentalidade; o contexto intrincado da relação com regulação da pesquisa, oportunidades de mercado, vulnerabilidade, disponibilidade de sujeitos experimentais e sobre como a participação em experimentos se tornou uma forma de medicina usual. Aborda também a possibilidade de produção de vieses nas pesquisas que buscam aprovação de medicamentos e a relação dessa experimentalidade com o direito à saúde.

As vendas de fármacos a nível global alcançaram \$712 bilhões em 2007. A América do Norte contabiliza mais da metade do total de vendas, e existe um acelerado crescimento em novos mercados da América Latina, Ásia, e Leste Europeu. De outro lado, em décadas recentes, têm alargado também o acesso a terapias experimentais, particularmente nas áreas da AIDS, câncer e tratamentos genéticos, e ambiciosos esforços federais e da indústria estão em curso para recrutar mais pessoas para ensaios clínicos nos Estados Unidos e exterior.

A autora apresenta o exemplo concreto de uma empresa que explora o campo dos experimentos com medicamentos. A empresa iniciou seus negócios em meados da década de 90 e tem crescido desde então. Ela tem uma dúzia de estabelecimentos de pesquisa afiliados, localizados em diversos contextos sócio-econômicos e cidades nos Estados Unidos, no Leste da Europa e Eurásia que executam todas as fases de ensaios clínicos. Médicos das Regiões do Leste Europeu e Eurásia estão dispostos a se tornar monitores de experimentos, fornecendo suporte para outros médicosinvestigadores e assegurando que os protocolos de pesquisa sejam corretamente executados. Os médicos dessas regiões são conhecidos como competentes, mas como mal remunerados e essas tarefas pagam consideravelmente mais que aquilo que um médico Russo recebe para tratar pacientes.

Além do exemplo dessa pequena companhia que foca somente em executar os experimentos apresenta o caso das Organizações Contratuais de Pesquisa (CROs) ${ }^{2}$, que oferecem um conjunto mais vasto de serviços para companhias farmacêuticas, biotecnológicas e de dispositivos médicos. Eles organizam e monitoram todos os estágios de experimentos globais multi-situados e guiam os seus clientes nos complexos ambientes regulatórios nacionais.

Refere também que há um crescente grupo de profissionais subcontratados que compõe a chamada indústria de ensaios clínicos, que facilita um estágio decisivo na ciência global de desenvolvimento de medicamento, cujo trabalho é parte de um empreendimento experimental em pleno desenvolvimento e expansão, com relação ao qual são largamente desconhecidos o escopo e as implicações mais amplas.

\footnotetext{
${ }^{1}$ Os ensaios clínicos são estudos designados para sistematicamente avaliar novas drogas ou novos usos em humanos de tratamentos conhecidos. Eles são um caminho para demonstrar o cumprimento dos requisitos da segurança e efetividade antes de os tratamentos ou medicamentos entrarem no mercado. PETRYNA, Adriana. When Experiments Travel: Clinical Trials and the Global Search For Human Subjects. Princeton University Press: Princeton, 2009, p. 1.

${ }^{2}$ Contract Research Organizations.
} 
Esses são alguns dos dados iniciais que nos mergulham nesse novo olhar que nos remete ao contexto da interação e do conturbado encontro entre o mercado, a inovação, e as necessidades de saúde pública.

\section{VARIABILIDADE ÉTICA}

No capítulo, em um primeiro momento, a autora dedica um subtópico à pergunta sobre o porquê de os ensaios clínicos estarem se globalizando. Uma das primeiras questões postas, por meio de entrevista a cientista corporativo que coordena ensaios clínicos é que, do ponto de vista desse profissional, existe uma desconfiança pública exagerada nos Estados Unidos e na Europa Ocidental e que isso está se tornando a maior barreira aos trabalhos. O profissional também expressa que se as pessoas não estão diante de uma situação de vida ou morte elas normalmente não estarão dispostas a participar de um ensaio clínico e que tanto os pesquisadores quanto os consumidores possuem uma dependência econômica sobre outras pessoas dispostas a dizer sim. Nesse sentido, o texto destaca que a experimentalidade envolve um tipo de relação de troca onde as pessoas com necessidades médicas não-atendidas estão dispostas a dizer sim para o movimento do capital global e produtos científicos e médicos.

No exemplo da Polônia, que possui uma das mais altas taxas de mortalidade relacionadas a doenças cardiovasculares no mundo, os hospitais públicos e o principal instituto do câncer se tornaram as destinações preferenciais para experimentos de terapias que vão de tratamentos para hipertensão a procedimentos cirúrgicos invasivos. Em conexão com isso há ponto de vista segundo o qual os ensaios clínicos seriam um bem social, uma parte integral da prestação de atendimento de saúde porque cada paciente que precise de tratamento o conseguiria de um ensaio clínico.

Com relação ao Brasil apresenta-se a crítica do crescente uso de ensaios clínicos como maneiras de introduzir medicamentos de alto custo no debilitado sistema público de saúde. "Após o término de um ensaio, a indústria recruta médicos para prescrever e pacientes para demandar medicamentos do estado”3

Os ensaios clínicos são geralmente divididos em quatro fazes. Nas três primeiras fazes são realizados testes com humanos, sendo que o número de participantes aumenta em cada uma delas, chegando a cerca de 1.000 - 3.000. Esses experimentos são coordenados através de múltiplos centros, os quais, cada vez mais, estão localizados mundialmente. A fase quatro consiste em estudos primariamente observacionais e não experimentais nos quais as companhias e reguladores confrontam informações sobre os riscos e benefícios uma vez que o medicamento entra no mercado.

Fatores como altos custos, resultados demorados, falta de financiamento, ônus regulatórios, infra-estruturar fragmentada são alguns dos problemas técnicos e regulatórios apontados. Nesse sentido aponta-se que a indústria de ensaios clínicos promete localizar e superar tais problemas e que desde 1980 as companhias farmacêuticas têm cada vez mais terceirizado a pesquisa clínica. O mercado das empresas contratuais de pesquisas (CRO) é estimado em \$10 bilhões e em crescimento.

${ }^{3}$ PETRYNA, Adriana. When Experiments Travel: Clinical Trials and the Global Search For Human Subjects. Princeton University Press: Princeton, 2009.Tradução nossa. p. 11. 
O texto também aponta que a geografia dos testes clínicos está mudando dramaticamente. Há um aumento do percentual de experimentos sendo realizados fora dos Estados Unidos. Verifica-se também o maior crescimento anual de investigadores ativos na Rússia, Argentina, Índia, Polônia, Brasil e China.

Como o mercado de ensaios clínicos se torna cada vez mais terceirizado e internacionalizado ${ }^{4}$, ele também se torna crescentemente descentralizado e mais difícil de localizar. As companhias são livres para mover a pesquisa médica, em escala global, para locais custo-efetivos. Entretanto, os sujeitos da pesquisa dependem das comunidades locais e nacionais para mediar a sua participação em ensaios clínicos e para proteger eles contra danos que possam estar associados a tais participações. A crescente "escolha" de se tornar sujeitos experimentais, feita por cidadãos de economias em transição, se relaciona com seu status de pobreza e frequentemente revela os limites dos padrões locais de atendimento e a falha dos estados em proteger seus cidadãos.

Também se percebe que existem debates e dúvidas sobre o grau de independência dessas empresas que são responsáveis pelo desenho dos experimentos. A respeito da detecção de danos os patrocinadores exercem significativo controle sobre a definição de um evento adverso reportável.

Para esse tipo de empresa o tempo e a rapidez são questões prementes. As CROs ${ }^{5}$ acessam os ambientes regulatórios dos países e verificam o tempo necessário para obter aprovação para iniciar os experimentos e também o tempo necessário para ter um estudo em andamento. Os termos analisados também identificam Países nos quais se deveria investir ou focar mais e Países nos quais as drogas sob estudo poderiam ser comercializáveis. Outro dado que a autora apresenta por meio de entrevista é que as companhias podem escolher populações para pegar o dado que precisam e obter um sinal mais claro dos benefícios dos medicamentos. Mas Adriana Petryna também apresenta depoimentos de profissionais enfatizando bons aspectos como, por exemplo, a produtividade e a transparência do empreendimento e também o trabalho dos monitores dos estudos, os quais revisam os registros dos experimentos para assegurar a observância dos protocolos e assegurar também que os dados estão "limpos", fazendo uma proteção do valor dos dados.

Em meio a economias em reestruturação e conflitos não resolvidos sobre o papel do Estado na regulação da economia e na prestação de atendimento de saúde ilhas de pesquisa clínica e mercados de novas drogas tomam forma. Nesse contexto são conhecidos problemas éticos que soam como "disponibilidade" de pacientes

\footnotetext{
${ }^{4}$ No original a expressão é offshored. O que nos remete tanto ao significado da expressão offshore que na terceira definição dada pelo Webster significaria "situado ou operando em um país estrangeiro" ("situated or operating in a foreign country <offshore mutual funds> <offshore banking>”). Disponível em <http://www.merriam-webster.com/dictionary/offshore>. Acesso em 31 ago. 2010. O termo também está relacionado com a questão das empresas offshore que são conhecidas por não conduzir os empreendimentos substancialmente em seus países. Em um âmbito geral essa conduta está ligada a benefícios, dentre os mais conhecidos estão a carga fiscal e o anonimato. Nesse sentido consultar:

<http://encyclopedia.thefreedictionary.com/Offshore+company> Acesso em 31 ago. 2010.

${ }^{5}$ Organizações Contratuais de Pesquisa.
} 
enfrentando crises médicas e pobreza, mas também existem outros menos evidentes como, apenas para citar um exemplo apontado pela autora, quem em última análise é responsável pelo bem-estar dos sujeitos experimentais a curto e longo prazo.

Petryna se debruça sobre a pergunta relativa ao que tem dirigido a demanda por um grupo mais amplo de sujeitos experimentais. Aponta como primeiro elemento o grande número de ensaios clínicos sendo executados. Comenta também a relação com o competitivo mercado das drogas me too, as quais, com uma mínima alteração farmacológica baseada ou imitando drogas campeãs de venda exploram mercados bem estabelecidos. O segundo elemento diz respeito às exigências regulatórias norte-americanas a respeito da segurança em longo prazo das novas drogas, sendo necessário incluir um maior número de pacientes nos experimentos. Aponta também o rápido crescimento de particulares classes terapêuticas e a corrida para ser o primeiro ter as drogas aprovadas e comercializáveis. Por último aponta que há significativo crescimento de novas entidades químicas e o fato de que patentes inundam o escritório norte-americano de patentes (U.S. Patent Office).

Outro fator importante tratado diz respeito ao fato de que os americanos e europeus ocidentais usam tantos medicamentos que estão se tornando inutilizáveis para testes com medicamentos devido à produção de interações entre drogas. Passando pelo escândalo da Talidomida, pela implementação dos testes fase quatro e pela adoção do ensaio randomizado controlado como forma de redução dos vieses, percebe-se que agora o viés pode ser induzido no desenho dos protocolos e no recrutamento de população conveniente.

As preocupações regulatórias e industriais sobre ética parecem ser matéria primariamente do nível de produção de dados, de assegurar a "integridade" dos dados. Em resumo, uma preocupação de que os dados produzidos em algum lugar do mundo possam cumprir as exigências para aprovação nos Estados Unidos. “Quando não enxergamos pacientes, enxergamos dados”, essa é uma expressão crítica reproduzida pela autora e que parece ter conexão com a questão.

A autora descobriu uma variabilidade ética na globalização dos experimentos, aponta que os padrões éticos internacionais falharam em tomar em conta os contextos locais e experiências vividas e se tornaram uma espécie de truísmo. Essa variabilidade ética evolui como uma tática que toma realidades de diferentes contextos locais e capacidades como um dado e como base na qual se consolida uma variabilidade do custo-efetividade da pesquisa com seres humanos.

Petryna apresenta o contexto relativo ao caso da controvérsia sobre o uso de placebo, na África em 1994, durante experimentos de curta duração com o tratamento AZT para parar a transmissão perinatal de HIV. Aponta que o caso é considerado um marco no debate sobre um padrão ético na pesquisa clínica global. O debate sobre o caso gerou a sexta revisão da declaração de Helsínquia de 1964. A revisão reiterou a posição contra o uso de placebo quando tratamentos padrões são conhecidos.

${ }^{6}$ PETRYNA, Adriana. When Experiments Travel: Clinical Trials and the Global Search For Human Subjects. Princeton University Press: Princeton, 2009.Tradução nossa. p. 17. 
Entretanto a leitura feita no $\mathrm{FDA}^{7}$ reduziu a significância regulatória da declaração ao apontar que a aceitabilidade dependeria do experimento e da população escolhida. E conforme declaração que colheu de analista de mercado ${ }^{8}$, o qual trabalhou em organização contratual de pesquisa, a saída adotada foi favorável ao que queria a indústria. Também, conforme ele, os pesquisadores poderiam ignorar o sombrio uso ético do placebo fornecendo o que é conhecido como "medicação equivalente" o que não significaria o tratamento melhor ou padrão, tornando factível que o paciente fique, na prática, sem tratamento. Então seria possível trabalhar a declaração, fazendo da ética um "documento funcional"’ . Dessa variabilidade ética passa-se ao complexo e complicado ambiente de evolução da indústria de pesquisa clínica.

\section{ARTE DO DESENVOLVIMENTO DE MEDICAMENTO}

Dentro do contexto das Organizações Contratuais de Pesquisa (CROs) apresenta-se a trajetória de profissional $^{10}$ que é tido como o fundador da indústria terceirizada de pesquisa clínica. Após o escândalo da Talidomida houve um endurecimento da regulação dos ensaios clínicos nos Estados Unidos. O profissional acompanhou esses acontecimentos e planejou sua preparação médica para se tornar um expert no desenvolvimento de medicamentos, mas na era regulatória Pós-Talidomida.

A Talidomida é amplamente conhecida por causar severos defeitos de nascimento. Sintetizada em 1953 pela companhia Chemie Gruenenthal da Alemanha Ocidental e colocada no Mercado em 1957, ela foi prescrita como uma pílula para dormir e também usada para tratar enjôos matinais em gestantes. No início dos anos 60 ela foi vendida ao redor do mundo. Em 1960 buscou-se a inserção no mercado Norte-Americano. Ao acaso pacientes relataram que o medicamento promovia uma boa noite de descanso, mas a Talidomida não tinha sido exaustivamente testada em animais e mulheres gestantes antes da comercialização. Cedo, relatórios de crianças com má-formação e em conexão com a Talidomida foram publicados nas maiores revistas médicas. Aproximadamente dez mil crianças em quarenta e seis países nascidas com deformidades induzidas pelo medicamento foram localizadas.

Percebe-se que após essa crise ficou muito mais difícil iniciar pesquisa nos Estados Unidos e principalmente para os estudos fase um. Então alguns estudos fase um e também fase dois cruzaram o oceano, dos EUA para a Europa.

A autora também apresenta a questão surgida no início dos anos 70 relativas à realização de testes em instituições como as prisões levando ao debate a respeito da existência de liberdade ou de coerção para que os presos participassem dos estudos.

Sujeitos experimentais têm sido obtidos rotineira e desproporcionalmente de grupos desfavorecidos e marginalizados. Muitos têm participado de pesquisa sem

\footnotetext{
${ }^{7}$ Food and Drug Administration.

${ }^{8}$ A expressão no original é market strategist.

${ }^{9}$ Ethics as "Workable Document". "Equivalent medication in Eastern Europe is not the same as equivalent medication in Western Europe, so you could work the Helsinki declaration”. PETRYNA, Adriana. When Experiments Travel: Clinical Trials and the Global Search For Human Subjects. Princeton University Press: Princeton, 2009. p. 36.

${ }^{10}$ Dr. Hein Besselaar.
} 
completo conhecimento e consentimento. Um dos exemplos apresentados é referente a uma pesquisa que começou em 1956, em que pesquisadores financiados pelo governo, por quatorze anos infectaram com o vírus da hepatite A, para observar o curso natural da hepatite infecciosa, crianças com problemas comportamentais internadas na Escola do Estado de Willowbrook em Nova York. Como havia um histórico de transmissão da doença no local os pesquisadores disseram que a atividade não gerava risco adicional e que seria melhor para as crianças serem infectadas sob condições controladas.

Em 1974 o Congresso aprovou norma ${ }^{11}$ para o estabelecimento de conselhos de revisão ${ }^{12}$ para avaliar a conduta ética de pesquisa e que autorizou a formação da Comissão Nacional para a Proteção de Sujeitos Humanos de Pesquisa Biomédica e Comportamental $^{13}$. A comissão publicou o relatório Belmont (Belmont Report), que sumarizou princípios éticos, que a autonomia individual fosse assegurada por procedimentos de consentimento informado, que a pesquisa devesse maximizar os benefícios e minimizar possíveis danos. Essas orientações foram revisadas e expandidas pela regulação federal, que estabeleceu níveis de proteção entre sujeitos vulneráveis e pesquisadores. Os prisioneiros, por exemplo, não poderiam ser sujeitos de experimentos não terapêuticos.

Em 1981, quando o Departamento de Saúde e Serviços Humanos e a Food and Drug Administration publicaram regulações embasadas no Relatório Belmont (The Belmon Report), as companhias farmacêuticas já tinham deslocado alguns de seus trabalhos fase um para outros países. E aquele profissional cuja trajetória a autora traça por meio de entrevista recebia muitas ofertas de trabalho, mas ele deixou a indústria farmacêutica e fundou sua própria firma de pesquisa ${ }^{14}$.

Adriana Petryna descreve o movimento da pesquisa, no início dos anos 90, dos centros de pesquisa acadêmicos para hospitais comuns, estabelecimentos de atendimento primário e estabelecimentos investigativos sem fins lucrativos, por conta das dificuldades enfrentadas nos hospitais acadêmicos.

No âmbito das Organizações Contratuais de Pesquisa (CROs) é apresentada a problemática de que, apesar dos custos crescentes, os pagamentos têm sido mantidos estáveis pela indústria farmacêutica, aproveitando-se do saturamento do mercado. E isso em conexão com o que é denominado de floater sites, sítios investigativos que prometem muitos pacientes, rotineiramente sob contratos baratos e que não estão meticulosamente comprometidos com a realização dos padrões de segurança e conformidade. Eles fazem o seu dinheiro e desaparecem. O escritório do FDA ${ }^{15}$ está recebendo um crescente número de queixas contra investigadores devido a violações de protocolos, falsificação de dados, não-observância do consentimento informado,

111974 National Research Act.

${ }^{12}$ Review boards.

${ }^{13}$ National Commission for Protection of Human Subjects of Biomedical and Behavioral Research.

${ }^{14}$ Como reproduz a autora, o Dr. Besselarr afirmou '“Gentlemen', we said, 'we are available to execute your study.” p. 68.

${ }^{15}$ FDA Offfice of Compliance. 
relatórios de eventos adversos insuficientes, e insuficientes prestações de contas dos medicamentos. Normalmente investigadores individuais são responsáveis, mas esses problemas são sistêmicos.

Conforme relato reproduzido por Petryna as $\mathrm{CROs}^{16}$ apenas seriam responsáveis pela execução do protocolo de estudo e normalmente supõe que o financiador do ensaio clínico tenha feito sua parte, mas algumas vezes preocupações éticas apenas surgem quando se pensa no que está sendo feito.

A autora entrevistou investigador que participou de uma equipe que conduziu estudos com uma droga retirada do mercado e ele relata que foi feita uma lista de sérios eventos adversos ${ }^{17}$, a qual foi enviada para o financiador do ensaio com recomendações. Mas o patrocinador evitou lidar com a informação e conseguiu-se a aprovação da droga. Percebe-se a grande dificuldade de se reportar eventos adversos porque o ensaio clínico como um todo pode ser fragilizado ou interrompido e também porque não se conseguirá novamente contratar com aquele cliente.

Nos últimos trinta anos a indústria alcançou um dos mais sustentáveis períodos de desenvolvimento científico, mas as descobertas científicas têm decaído desde o final dos anos oitenta. Os pseudo-arquivamentos sob a lei de patentes e os medicamentos mee-too ${ }^{18}$ são alguns dos expedientes utilizados pela indústria. A autora transcreve reclamações no sentido de que a indústria estaria pressionando baixa de custos com relação às $\mathrm{CROs}^{19}$, de que isso acabaria por pressionar também a qualidade e geraria o abandono do método científico. Em relação à globalização dos ensaios clínicos os experimentos podem trazer benefícios em curto prazo para comunidades locais, mas que no longo prazo são desconhecidos para o usuário final. Daí que os indivíduos sejam tornados vulneráveis em vários níveis.

Entretanto, a descentralização e globalização dos ensaios clínicos oferecem uma mobilidade sem precedentes e ficam ressaltadas as distorções.

\section{O ENSAIO CLÍNICO GLOBAL}

Não se sabe quantos ensaios clínicos estão sendo realizados ao redor do mundo atualmente e há um esforço da Organização Mundial de Saúde (OMS) para criar um banco de dados global de ensaios clínicos. Um banco de dados global pode ajudar a redefinir prioridades de pesquisa, reduzir duplicação de esforços de pesquisa e assegurar transparência. Os órgãos regulatórios poderiam delinear, a partir dos registros, melhores objetivos de saúde pública. Poderiam também aperfeiçoar a fiscalização da pesquisa e mecanismos de proteção das pessoas. A indústria contesta o monitoramento da pesquisa, em suma, porque a divulgação de informações poderia trazer desvantagens competitivas em relação aos rivais. Entretanto os críticos dessa posição apontam que normalmente as companhias sabem o que as outras desenvolvem e que isso não atrapalharia a competitividade se todas as empresas estivessem obrigadas.

\footnotetext{
${ }^{16}$ Organizações Contratuais de Pesquisa.

${ }^{17}$ Serious Adverse Events (SAEs).

${ }^{18}$ Com uma mínima alteração farmacológica baseada ou imitando drogas campeãs de venda exploram mercados bem estabelecidos.

${ }^{19}$ Organizações Contratuais de Pesquisa.
} 
Após expor sobre a Polônia, que teve uma das mais altas taxas do mundo de mortalidade devido a ataque cardíaco, um país que inicialmente foi chave para os ensaios clínicos internacionalizados ${ }^{20}$ e que dentre as caracteríscas positivas conta com o excepcional nível de qualidade dos dados que produz, a autora aborda sobre uma empresa subcontratada situada no sudeste europeu, que, segundo entrevista, possui um sítio de experimentos que nunca tinha sido auditado pelo FDA ${ }^{21}$. Há uma busca por expandir os ensaios clínicos para além dos ambientes mais padronizados, regulados e caros. Isso parece estar conectado com o dado segundo o qual houve um declínio do mercado de ensaios clínicos polonês em meados de 2006. Isso em razão de ser reputada como impositora de excessivos obstáculos regulamentares e fiscais ${ }^{22}$.

Os experimentos podem estabilizar em um país, mas em determinado momento podem mudar para outro lugar. Um exemplo apresentado é o da República Checa, quando baniu o uso de placebo a indústria moveu seus investimentos, quando voltou atrás sobre isso a indústria retornou. As companhias recompensam com mais investimentos os países que facilitam as pesquisas e as vendas. Entretanto alguns países têm um papel mais importante que outros na estratégia de estabelecer o mais alto preço precedente. Nos Estados Unidos não há controle de preços, por isso pode-se conseguir o mais alto valor, mas a indústria também busca orquestrar seu enfoque para maximizar o preço também nos mercados regionais.

De um lado, críticos do crescimento dos ensaios clínicos nos países em desenvolvimento têm apontado a questão da coerção, a falta de uma participação voluntária e informada, e um inadequado consentimento informado como os problemas mais comuns. Agentes da indústria e alguns médicos, por outro lado, tendem a promover os ensaios clínicos como um bem social em contextos improvisados, enfatizando que a participação possibilita acesso a tratamentos não disponíveis.

A autora, por meio de entrevista, percebe mais claramente como ideias de uma abordagem paciente-consumidor direcionam o recrutamento, levando a questões sobre a integridade do processo de consentimento informado. O entrevistado, inclusive, fornece um exemplo de uma estratégia de recrutamento “empreendedor", como recrutar pacientes não tratados para um estudo placedo controlado, mas de uma maneira ética. A autora narra que ele achou a abordagem brilhante, mas que ela achou preocupante. E a sequência de operações seria a seguinte: "Nós todos sabemos que é antiético retirar tratamento de pacientes durante um experimento. Se pacientes podem obter o tratamento necessário onde eles vivem, então nós certamente não podemos reter tratamento ou usar um placebo"23. "Considerando um paciente com diagnóstico recente, diga-se hipertensão, reter o tratamento é eticamente

\footnotetext{
${ }^{20}$ No original a expressão é offshored clinical trials. Consultar nota de rodapé número 6 .

${ }^{21}$ Foods and Drugs Administration.

${ }^{22}$ p. 100, ver também p. 107-109 sobre a International Conference on Harmonisation of Technical Requeriments for Registration of Pharmaceuticals for Human Use (ICH) e o Global Clinical Practice $(G C P)$. Ambos unificaram padrões na EU, EUA e Japan, o que inclui a Polônia.

${ }^{23}$ PETRYNA, Adriana. When Experiments Travel: Clinical Trials and the Global Search For Human Subjects. Princeton University Press: Princeton, 2009. p. 125. Tradução nossa.
} 
justificável."24 A autora pergunta como e o entrevistado responde que seria totalmente aceitável esperar e ver se a hipertensão do paciente pode ser controlada por meios não farmacêuticos (dieta, menos sal, exercício). Outro elemento importante é que muitas vezes a participação nos testes com placebo são aceitos devido à expectativa de, após, receber o tratamento. E o próprio recrutamento passa por um processo de diagnóstico que é apresentado ao paciente como um presente e também frente à possibilidade de acesso a tratamentos caros.

O processo de recrutamento nessa instância surge com a criação de um paciente-consumidor que compra uma ideia particular da doença, que é educado pelo recrutador sobre o melhor tratamento possível e que percebe que ele é incapaz de obter aquele atendimento fora de um experimento. O momento da compra é o momento do consentimento informado e esse consentimento é um pouco diferente do consentimento tradicional, onde a autonomia fica de alguma forma mantida, mas também não é exatamente coerção, visto que é combinado com o oferecimento de bens médicos (atendimento, medicamentos, etc.).

A seguir, o que se percebe principalmente é que a experimentalidade afeta e adentra os sistemas públicos de saúde, criando novas formas de resposta, inclusive na avaliação de tecnologia.

\section{FÁRMACOS E O DIREITO À SAÚDE}

Adriana Petryna mostra que, enquanto os dados dos experimentos têm sido enviados para as sedes das corporações, novas necessidades médicas surgem localmente. Pacientes passam a requerer tratamentos que estão fora de seu alcance. Existem também crescentes demandas impostas por pacientes e comercializadores sobre os médicos para prescrever e sobre o estado para fornecer medicamentos novos de alto custo e para os quais os benefícios clínicos não estão unequivocamente estabelecidos.

Nesse capítulo a autora analisa, em grande parte, o contexto brasileiro, a começar pela situação de aumento de processos judiciais movidos pelos cidadãos contra o estado com o objetivo de obrigá-lo a fornecer medicamentos de alto custo.

Especialista aponta o problema referente à prescrição dos mais novos medicamentos enquanto muitos problemas poderiam ser tratados com um ou dois medicamentos antigos e aponta que os pacientes são inundados com medicamentos mee too $^{25}$. Por outro lado, a autora também apresenta o caso da mãe de um paciente que possuía uma doença rara (Gaucher) e que teve de recorrer ao judiciário para obter o medicamento de alto custo, o qual acabou sendo incorporado no sistema público em 1995.

Sobre a avaliação de tecnologia de saúde no Brasil apresenta o movimento que culminou na publicação dos Protocolos Clínicos e Diretrizes Terapêuticas: Medicamentos Excepcionais, que possui objetivo de estabelecer um mecanismo para garantir a segurança e eficácia da prescrição. O manual de Protocolos continha 31

${ }^{24}$ PETRYNA, Adriana. When Experiments Travel: Clinical Trials and the Global Search For Human Subjects. Princeton University Press: Princeton, 2009. p. 125. Tradução nossa.

${ }^{25}$ Consultar nota de rodapé número 19. 
protocolos clínicos e diretrizes terapêuticas para prescrição de medicamentos excepcionais, que passaram por uma consulta pública. Os Protocolos revisam a situação clínica a ser tratada e a classificação da doença e apresentam critérios de diagnóstico assim como para inclusão e exclusão de tratamento.

Referente à judicialização aponta que a litigância sobre o direito à saúde é tecida de muitos tópicos diferentes, incluindo mobilização social ancorada em longas histórias de exclusão de cidadãos dos mecanismos legais formais, sistemas de saúde frágeis, juízes medicalmente desinformados e uma “judicialização” geral das políticas.

Menos que cinco por cento dos estudos publicados em revistas médicas são válidos e relevantes para os clínicos e administradores públicos e quando novos medicamentos são lançados em mercados emergentes como Brasil e Polônia essa desconexão pode aumentar e gerar novos tipos de problemas de saúde pública. Observa-se que se sabe que muitos novos medicamentos não matam, mas que não se sabe também se podem salvar e o quão custo-efetivos são.

A autora apresenta o trabalho realizado pela equipe de Dr. Picon e pela Unidade de Pesquisa Clínica. Um trabalho que inclui a análise do planejamento de ensaios clínicos, verificando falhas nos dados e identificando aspectos subestudados. Nesse sentido apresenta-se o caso referente ao medicamento Vioxx cuja incorporação ao sistema público foi negada, e também o caso referente ao tratamento da doença de Gaucher, cuja dosagem do tratamento foi diminuída em razão de se ter verificado que a indicada pela indústria era maior do que a necessária para uma ótima resposta. Ambos os casos sofreram grande reação por parte de setores médicos, em um contexto que sugere a existência de conflitos de interesse.

A Unidade de Pesquisa Clínica foi aberta em 2003 e possui equipamentos financiados pela ANVISA. Desde 1999 a ANVISA tem fundado unidades nas maiores universidades com o objetivo de realizar testes para a aprovação de medicamentos genéricos. A unidade também foi pensada para atrair ensaios financiados pela indústria. O ambiente de negócios favorável do Brasil, médicos bem treinados e o crescimento do mercado farmacêutico têm feito do país uma destinação atrativa para os ensaios clínicos. A gerente financeira da unidade fala que o governo federal tem diminuído investimento em pesquisa e infra-estrutura médica e que os hospitais universitários estão lutando para encontrar fontes alternativas de financiamento.

Dr. Picon aponta que para a realização de testes de medicamentos genéricos foi estabelecida uma parceria com a Faculdade de Farmácia da Universidade Federal do Rio Grande do Sul com o desafio de reverter duas tendências: as companhias estavam testando os genéricos em relação ao tratamento padrão dentro de suas próprias empresas e a maioria dos estudos clínicos ainda eram realizados em ambulatórios. A responsabilidade pública estava em jogo e os ambientes investigacionais devem estar livres de conflitos de interesse.

Contudo os testes com medicamentos genéricos nunca decolaram realmente na Unidade de Pesquisa Clínica. Entretanto, continua desempenhando um importante papel na administração do hospital para reformular sua estratégia de pesquisa clínica. 
Foi central nos esforços de Dr. Picon para a implementação dos Protocolos e agora Dr. Picon está criando, dentro e fora do hospital, uma rede de centros de referência para doenças. Esses Centros de Referência reuniriam dados dos pacientes e sobre a eficácia e efetividade de algumas das diretrizes e a unidade seria uma base operacional para planejamento. O primeiro Centro de Referência criado foi o relativo à doença de Gaucher. Inicialmente o tratamento para a doença não foi provido pelo estado, mas com a criação do Centro de Referência as famílias, que haviam se mobilizado para obtê-lo, finalmente retomaram suas vidas e suas rotinas normais.

Na obra também surge o questionamento sobre o que acontece quando os ensaios clínicos terminam. O Brasil é uma importante local para teste de terapias genéticas não somente pela competência técnica na área, mas também por conta de seu sistema de saúde universal. Nesse sentido, percebe-se que pacientes participam de ensaios clínicos na perspectiva de receber tratamento, mas o problema inicia quando os testes terminam. A indústria continua o fornecimento por um tempo, mas isso é uma decisão discricionária da companhia, inclusive a autora reproduz relato de profissional no sentido de que já ouviu que representantes da indústria orientam pacientes a mover ações para acessar novos medicamentos. E relata-se que é muito difícil colocar nos contratos uma cláusula que garanta a continuidade do tratamento. Nesse sentido a sugestão que vários médicos dão é a de que deveria haver uma proteção por parte da legislação: se uma pessoa muito doente participar de estudo e ficarem bem estabelecidos os benefícios do medicamento então ele deveria receber o tratamento durante tempo necessário.

Inclusive a autora traz o exemplo de um estudo fase três de uma nova terapia para uma desordem genética relacionada à Gaucher o qual, para o desalento dos médicos e dos pacientes, foi interrompido no terceiro ano.

Quando a terapia de reposição enzimática funcionou para a doença de Gaucher todos começaram a prestar atenção para outras desordens de deficiência enzimática. Entretanto a evidência, nesse caso, não é tão bem estabelecida quanto a estabelecido para a doença de Gaucher. De outro lado o medicamento havia diminuído a progressão da falência renal em vários pacientes e se o tratamento fosse interrompido a perda de função renal reiniciaria. Pessoas conscientes da retirada do tratamento suspeitaram que a companhia simplesmente quisesse o reembolso do estudo, que agora era entendido como tratamento. Os sujeitos experimentais estavam efetivamente instruídos sobre as especificidades dos seus direitos à saúde. Alguns pacientes migraram para unidades de pesquisa em outros estados; alguns moveram ações contra o Estado do Rio Grande do Sul; e uns poucos ligavam semanalmente perguntando se o tratamento estava disponível.

Petryna contextualiza com a apresentação de um caso concreto. Um dos pacientes que participava do estudo recebeu contato de advogado e foi iniciada uma ação judicial, a qual recebeu uma primeira decisão favorável que posteriormente foi anulada pela corte estadual superior e estava sendo levada para a suprema corte.

Algumas das questões que se percebe no texto e nos relatos transcritos pela autora são: toda a discussão sobre o alto custo do tratamento e as pressões pelo 
fornecimento de medicamentos caros, a falta de opção e as dúvidas do paciente sobre sua melhora com o uso do medicamento, um clima de desconfiança que leva o paciente a declarar que não é parte de um esquema de corrupção, a iniciativa da administração do hospital estimulando a companhia a reiniciar o fluxo do estudo (o que acabou fazendo) e pesquisadores contatando outras companhias que testavam terapias similares. Esse ambiente conturbado sugere uma assimetria de informação e atuação.

Segundo a autora esse caso mostra o limite onde a política falha em conhecer a extensão dos riscos que acompanham esse modelo global de negócio experimental e que as instituições públicas têm um papel vital arbitrando a lógica de mercado, resolvendo as "impossibilidades” que pacientes e investigadores bem-intencionados encontram, e garantindo o acesso dos cidadãos a novos medicamentos.

\section{CONCLUSÃO}

Em suas conclusões Petryna aponta que a pesquisa clínica é agora uma empresa mundial de fabricação de dados, que tem crescido em paralelo com os critérios para aprovação de medicamentos e incentivos, fiscalização regulatória, evolução das regras comerciais, e oportunidades de negócios relacionadas. A autora questiona a adequação das normas de proteção nos Estados Unidos e internacionalmente, como elas variam de lugar para lugar, e como os dados são estrategicamente manufaturados e, às vezes, estrategicamente retirados.

Para a autora são necessárias medidas no nível nacional e internacional que assegurem aos sujeitos experimentais algum benefício (na forma de tratamento pós-experimento) e para assegurar que os consumidores não se tornem extensões experimentais não voluntárias de ensaios clínicos que possibilitam a inserção de drogas inefetivas no mercado.

Aparentemente relacionado com o trabalho que é realizado na Unidade de Pesquisa Clínica, entende que novos programas de avaliação de tecnologia que possam independentemente acessar o valor e a inovatividade de novas tecnologias médicas precisam ser reforçadas para que as instituições de saúde pública, as quais enfrentam dificuldades financeiras, tenham uma chance razoável de promover responsável e sustentavelmente o atendimento de saúde, no lugar de sucumbir a uma forma de atendimento farmaceuticalizada.

A experimentalidade subscreve uma agenda de pesquisa que não necessariamente provê os mais válidos e relevantes resultados médicos e ainda introduz novos riscos. Aponta que gastos massivos em prescrição de medicamentos sobrecarregam os provedores públicos de atendimento de saúde. No Brasil, em conexão com a medicina direcionada pelo mercado, reformas neoliberais do estado, e discursos de direitos humanos, vislumbra que os cidadãos são reduzidos no seu próprio hiper-individualizado conflito por tratamento. 